\title{
Accord trouvé entre les médecins valaisans et la CSS Assurance
}

Lors d'une séance de médiation organisée par le Département de la santé (DSSE), un accord a été trouvé entre la Société Médicale du Valais (SMV) et la CSS Assurance. Cet accord concerne I'assurance alternative «Profit» de la CSS. Les médecins spécialistes qui assument un service de garde régulier figureront désormais sur la liste «Profit». Le service de garde se poursuivra donc en Valais et le conflit entre les médecins et la CSS est ainsi réglé.

Présidence du Conseil d'Etat, Information, le chef de l'information de l'Etat du Valais
Correspondance:

Palais du Gouvernement

Case postale

CH-1951 Sion

Tél. 0276062095

Fax 0276062094

bernard.reist@admin.vs.ch
Date: 13 septembre 2006

L'assurance alternative «Profit» est un modèle du médecin de famille qui vise à renforcer la position des médecins qui fournissent les soins de base et à récompenser les clients qui adoptent un comportement d'économie. Les médecins qui sont admis dans le modèle «Profit» sont des médecins de médecine générale, de médecine interne, de pédiatrie ainsi que des médecins omnipraticiens. Les assurés de la CSS qui choisissent l'assurance «Profit» bénéficient d'un rabais de $8 \%$ sur la prime de l'assurance de base. En contrepartie, ils doivent consulter, en premier lieu, un médecin désigné qui figure sur la liste «Profit».

La SMV demandait par le passé que tous les médecins, y compris les spécialistes qui assumaient un service de garde sur mandat de la SMV, soient admis dans la liste «Profit». Elle partait du principe que de nombreux spécialistes en Valais exerçaient également une activité de généraliste en complément de leur spécialisation.

\section{Accord entre la SMV et la cSS}

Le Chef du Département de la santé a réuni, le 13 septembre 2006, les partenaires pour une ultime médiation. Lors de cette rencontre, les discussions entre la CSS et la Société Médicale du Valais ont abouti à l'accord suivant:

- La CSS admet dans la liste «Profit» les spécialistes qui, sur mandat de la SMV, assument un service de garde régulier et de manière équivalente aux médecins de $1^{\text {er }}$ recours.

- La SMV se met à disposition de la CSS pour tenir à jour la liste «Profit». Il s'agit de signaler les médecins spécialistes qui assument un service de garde régulier. Les médecins, dont la commission paritaire (médecins/assureurs) aura constaté le non respect des critères d'économicité ne figureront pas sur la liste.

- Après une année, les parties feront le point de la situation au cours d'une réunion organisée par le Département de la santé du canton du Valais.

Grâce à l'accord trouvé entre la SMV et la CSS, les différends sont réglés. Les deux parties sont satisfaites de cet accord. 